\title{
$A b$ initio construction of the energy density functional for electron systems with the functional-renormalization-group-aided density functional theory
}

\author{
Takeru Yokota $\odot^{1, *}$ and Tomoya Naito $\odot^{2,3, \uparrow}$ \\ ${ }^{1}$ Institute for Solid State Physics, The University of Tokyo, Kashiwa, Chiba 277-8581, Japan \\ ${ }^{2}$ Department of Physics, Graduate School of Science, The University of Tokyo, Tokyo 113-0033, Japan \\ ${ }^{3}$ RIKEN Nishina Center, Wako 351-0198, Japan
}

(Received 23 October 2020; accepted 25 January 2021; published 16 February 2021)

\begin{abstract}
We show an ab initio construction of the energy density functional (EDF) for electron systems using the functional renormalization group. The correlation energies of the homogeneous electron gas given in our framework reproduce the exact behavior at high density and agree with the Monte Carlo data in a wide range of densities. Our analytic technique enables us to get the correlation energies efficiently for various densities, which realizes the determination of EDF in the local density approximation (LDA) without any fitting for physically relevant densities. Applied to the Kohn-Sham calculation for the noble gas atoms, our EDF shows comparable results to those of other conventional ones in LDA.
\end{abstract}

DOI: 10.1103/PhysRevResearch.3.L012015

Introduction. Density functional theory (DFT) [1] is a successful framework to analyze quantum many-body systems providing an efficient way known as the Kohn-Sham (KS) scheme [2], and has been employed in various fields, including condensed matter physics, quantum chemistry, and nuclear physics. DFT is often positioned as a first-principles method. However, most of the energy density functionals (EDFs), which govern the accuracy of DFT calculations, are empirically constructed, and the recipe to systematically construct EDF based on microscopic Hamiltonians has not been established yet [3-5].

In this Letter, we focus on an attempt for the microscopic construction of the EDF put forward in Refs. [6,7], which we call the functional-renormalization-group-aided DFT (FRGDFT). This is based on the functional renormalization group (FRG) [8-11] (for reviews, see, e.g., Refs. [12-16]), which is an established method for quantum many-body systems. In FRG, a one-parameter exact flow equation for the effective action, the quantum counterpart of the classical action, is utilized to nonperturbatively include quantum or thermal fluctuations. Owing to the fact that the EDF can be defined by the effective action $\Gamma[\rho]$ with the local density $\rho$ [17-19], accumulated methods in FRG are expected to be applied to the construction of EDF.

Applications of the FRG-DFT accomplished recently include analysis of the ground states in lower-than- $(1+1)$ dimensional systems [20-23] and (2+1)-dimensional

\footnotetext{
*tyokota@issp.u-tokyo.ac.jp

†tomoya.naito@ riken.jp

Published by the American Physical Society under the terms of the Creative Commons Attribution 4.0 International license. Further distribution of this work must maintain attribution to the author(s) and the published article's title, journal citation, and DOI.
}

homogeneous electron gas [24], excited states of a $(1+1)$ dimensional systems [25], and formalism for superfluid systems [26]. However, there has been no numerical application to the $(3+1)$-dimensional systems yet, which must be achieved to establish the FRG-DFT as a practical method. In particular, as systems for which DFT is frequently employed, the electron systems are one of the most important targets.

The aim of this Letter is the microscopic derivation of the $\operatorname{EDF} E[\rho]$ for the $(3+1)$-dimensional spin-unpolarized electron systems with the aid of the FRG-DFT. As a first step of the microscopic construction of the EDF, we consider the exchange-correlation part in the local density approximation (LDA) and aim at the construction of the correlation part. To this end, we apply the FRG-DFT to the $(3+1)$-dimensional homogeneous electron gas (3DHEG), derive the expression for the correlation energy per particle $\varepsilon_{\text {corr }}$ by solving the flow equation analytically by employing the second-order vertex expansion, and obtain $\varepsilon_{\text {corr }}\left(r_{\mathrm{s}}\right)$ as a function of the WignerSeitz radius $r_{\mathrm{s}}=[3 /(4 \pi \rho)]^{1 / 3}$. Our $\varepsilon_{\text {corr }}\left(r_{\mathrm{s}}\right)$ reproduces the exact behavior at the high-density limit given by the result of Gell-Mann-Brueckner resummation $\varepsilon_{\mathrm{corr}}^{\mathrm{GB}}\left(r_{\mathrm{s}}\right)$ [27] and agrees with the results of the diffusion Monte Carlo (DMC) calculations [28-30] in a wide range of densities.

A concern about the application to $(3+1)$-dimensional systems may be that the coordinate or momentum integrals in the FRG-DFT calculation become time consuming. For 3DHEG, however, we find that the dimension of the integrals can be drastically reduced with analytic techniques, which enables us to obtain $\varepsilon_{\text {corr }}\left(r_{\mathrm{s}}\right)$ densely enough to determine the EDF without fitting for physically relevant densities. This is in contrast to other conventional LDA EDFs, most of which are determined based on empirical choice of the fitting function for a few DMC data points.

Furthermore, applying the KS calculation of the ground states of the noble gas atoms, we demonstrate that the EDF 
constructed from our FRG-DFT data shows comparable results to other conventional LDA EDFs.

In this Letter, Hartree atomic units are employed.

$F R G-D F T$. We briefly summarize the formalism of FRGDFT and our analytic results in the 3DHEG, where electrons and background ions neutralizing the system interact with each other via the two-body Coulomb interaction $U(x)=$ $1 /|\boldsymbol{x}|$. Following Refs. [6,7], we consider the evolution when the interparticle interaction is gradually turned on. Let us employ a parameterized two-body interaction $U_{\lambda}(\boldsymbol{x})$ with the evolution parameter $\lambda$ running from $\lambda=0$ to 1 , and set $U_{\lambda=0}(\boldsymbol{x})=0$ and $U_{\lambda=1}(\boldsymbol{x})=U(\boldsymbol{x})$ to describe the evolution from the free to the fully interacting systems. Not only the electron-electron but also the electron-ion and ion-ion interactions are substituted by $U_{\lambda}(\boldsymbol{x})$ so as to keep the system neutral and avoid divergence caused by the Hartree energy during the evolution [24].

The key quantity of the FRG-DFT is the effective action $\Gamma_{\lambda}[\rho]$ for density $\rho$. To define it, we start from the action depending on $\lambda$ in the imaginary-time formalism:

$$
\begin{aligned}
S_{\lambda}\left[\psi, \psi^{\dagger}\right]= & \int_{X} \psi^{\dagger}\left(X_{\varepsilon}\right)\left(\partial_{\tau}-\frac{\nabla^{2}}{2}\right) \psi(X) \\
& +\frac{1}{2} \int_{X, X^{\prime}} U_{2 \mathrm{~b}, \lambda}\left(X, X^{\prime}\right) \hat{\rho}_{\Delta}(X) \hat{\rho}_{\Delta}\left(X^{\prime}\right) .
\end{aligned}
$$

Here, we have introduced $X=(\tau, \boldsymbol{x})$ and $\int_{X}=$ $\int d \tau \int d \boldsymbol{x}$ with imaginary time $\tau$ and spatial coordinate $\quad \boldsymbol{x}, \quad U_{2 \mathrm{~b}, \lambda}\left(X, X^{\prime}\right)=\delta\left(\tau-\tau^{\prime}\right) U_{\lambda}\left(\boldsymbol{x}-\boldsymbol{x}^{\prime}\right), \quad \psi(X)=$ ${ }^{\mathrm{t}}\left[\psi_{\uparrow}(X), \psi_{\downarrow}(X)\right]$ standing for the electron field with spin $\uparrow$ and $\downarrow$, and $\hat{\rho}_{\Delta}(X)=\hat{\rho}(X)-n_{e}$ with the density field $\hat{\rho}(X)=\psi^{\dagger}\left(X_{\varepsilon}\right) \psi(X)$ and $n_{e}=3 /\left(4 \pi r_{\mathrm{s}}^{3}\right)$ being the densities of electrons and background ions. The second term includes the electron-electron, electron-ion, and ion-ion interaction terms. We have also introduced $X_{\varepsilon}=(\tau+\varepsilon, \boldsymbol{x})$ with an infinitesimal $\varepsilon>0$ so that the Hamiltonian corresponding to $S_{\lambda}$ becomes normal ordered [23]. Then, $S_{\lambda}$ defines $\Gamma_{\lambda}[\rho]$ as

$$
\Gamma_{\lambda}[\rho]=\sup _{J}\left(\int_{X} J(X) \rho(X)-\ln Z_{\lambda}[J]\right),
$$

where

$$
Z_{\lambda}[J]=\int \mathcal{D} \psi \mathcal{D} \psi^{\dagger} e^{-S_{\lambda}\left[\psi, \psi^{\dagger}\right]+\int_{X} J(X) \hat{\rho}(X)}
$$

is the generating functional for density correlation functions and $\rho(X)$ is an arbitrary density. A notable feature of $\Gamma_{\lambda}[\rho]$ is that it satisfies the variational principle and gives the groundstate energy and density $[17,19]$, which means that the EDF $E_{\lambda}[\rho]$ is identified with $\Gamma_{\lambda}[\rho]$ as $E_{\lambda}[\rho]=\lim _{\beta \rightarrow \infty} \Gamma_{\lambda}[\rho] / \beta$ with the inverse temperature $\beta=\int d \tau$.

The key equation in the FRG-DFT is the evolution equation determining $\Gamma_{\lambda}[\rho][7,20,21,23,24]$ :

$$
\begin{aligned}
\partial_{\lambda} \Gamma_{\lambda}[\rho]= & \frac{1}{2} \int_{X, X^{\prime}} \partial_{\lambda} U_{2 \mathrm{~b}, \lambda}\left(X, X^{\prime}\right)\left[\rho_{\Delta}(X) \rho_{\Delta}\left(X^{\prime}\right)\right. \\
& \left.+\Gamma_{\lambda}^{(2)-1}[\rho]\left(X_{\varepsilon^{\prime}}, X^{\prime}\right)-\rho(X) \delta\left(\boldsymbol{x}-\boldsymbol{x}^{\prime}\right)\right],
\end{aligned}
$$

where $\rho_{\Delta}(X)=\rho(X)-n_{e}$ and $\Gamma_{\lambda}^{(2)-1}[\rho]\left(X, X^{\prime}\right)$ being the inverse of $\frac{\delta^{2} \Gamma_{\lambda}[\rho]}{\delta \rho(X) \delta \rho\left(X^{\prime}\right)}$, which satisfies

$$
\int_{X^{\prime \prime}} \Gamma_{\lambda}^{(2)-1}[\rho]\left(X, X^{\prime \prime}\right) \frac{\delta^{2} \Gamma_{\lambda}[\rho]}{\delta \rho\left(X^{\prime \prime}\right) \delta \rho\left(X^{\prime}\right)}=\delta\left(X-X^{\prime}\right) .
$$

Also, $X_{\varepsilon^{\prime}}$ is defined in the same manner as $X_{\varepsilon}$, but $\varepsilon^{\prime} \rightarrow 0$ limit is taken after $\varepsilon \rightarrow 0$ so that $\Gamma_{\lambda}^{(2)-1}[\rho]\left(X_{\varepsilon^{\prime}}, X^{\prime}\right)$ can be treated as the density correlation function [23]. The crucial point of Eq. (1) is that it is written in a closed form of $\Gamma_{\lambda}[\rho]$, which provides systematic schemes for the derivation of $\Gamma_{\lambda}[\rho]$.

Practically, the functional differential equation [Eq. (1)] needs to be converted to some numerically solvable equations. Here, we introduce the vertex expansion [6,7]: The functional Taylor expansion around a homogeneous density $\rho(X)=n_{e}$ is applied to Eq. (1), which yields a hierarchy of differential equations for density correlation functions [21,23-25]. We consider the expansion up to the second order and truncate higher-order terms. The equations up to the second order in the momentum-space representation read

$$
\begin{aligned}
\partial_{\lambda} \varepsilon_{\mathrm{gs}, \lambda} & =\frac{1}{2 n_{e}} \int_{\boldsymbol{p}} \partial_{\lambda} \tilde{U}_{\lambda}(\boldsymbol{p})\left[\int_{\omega} e^{i \omega \varepsilon^{\prime}} \tilde{G}_{\lambda}^{(2)}(P)-n_{e}\right], \\
\partial_{\lambda} \tilde{G}_{\lambda}^{(2)}(P) & =-\partial_{\lambda} \tilde{U}_{\lambda}(\boldsymbol{p})\left[\tilde{G}_{\lambda}^{(2)}(P)\right]^{2}+C_{\lambda}(P),
\end{aligned}
$$

where $\tilde{U}_{\lambda}(\boldsymbol{p})$ is the Fourier transform of $U_{\lambda}(\boldsymbol{x})$ and $\varepsilon_{\mathrm{gs}, \lambda}=$ $\lim _{\beta \rightarrow \infty} \Gamma_{\lambda}\left[n_{e}\right] /(\beta N)$ with $N=n_{e} \int d \boldsymbol{x}$ being the total particle number is the ground-state energy per particle. Here, we have introduced $P=(\omega, \boldsymbol{p})$ and $\int_{P}=\int_{\omega} \int_{\boldsymbol{p}}=$ $\int d \omega /(2 \pi) \int d \boldsymbol{p} /(2 \pi)^{3}$ with the Matsubara frequency $\omega$ and the spatial momentum $\boldsymbol{p}$,

$$
\begin{aligned}
C_{\lambda}(P)= & -\frac{1}{2} \int_{P^{\prime}} \partial_{\lambda} \tilde{U}_{\lambda}\left(\boldsymbol{p}^{\prime}\right)\left[\tilde{G}_{\lambda}^{(4)}\left(P^{\prime},-P^{\prime}, P\right)\right. \\
& \left.-\tilde{G}_{\lambda}^{(2)}(0)^{-1} \tilde{G}_{\lambda}^{(3)}\left(P^{\prime},-P^{\prime}\right) \tilde{G}_{\lambda}^{(3)}(P,-P)\right],
\end{aligned}
$$

and $\tilde{G}_{\lambda}^{(n)}\left(P_{1}, \ldots, P_{n-1}\right)$ being the connected density correlation function. Since $C_{\lambda}(P)$ is composed of higher-order correlation functions, an approximation for it is required. Here, we employ the approximation $C_{\lambda}(P) \approx C_{\lambda=0}(P)$, with which Eqs. (2) and (3) can be solved analytically when $U_{\lambda}(\boldsymbol{x})$ is chosen as $U_{\lambda}(\boldsymbol{x})=\lambda U_{\lambda=1}(\boldsymbol{x})$ [24].

Extracting $\varepsilon_{\mathrm{corr}}$ from $\varepsilon_{\mathrm{gs}, \lambda=1}$, we obtain

$$
\varepsilon_{\text {corr }}\left(r_{\mathrm{s}}\right)=\frac{1}{2 n_{e}} \int_{P}\left[\ln f\left(A_{P}, B_{P}\right)-A_{P}\right],
$$

which plays the central role in our construction of the EDF. Here, we have introduced $f(x, y)=\cosh y+(x / y) \sinh y$, $A_{P}:=\tilde{U}(\boldsymbol{p}) \tilde{G}_{\lambda=0}^{(2)}(P)$, and $B_{P}:=\left[\tilde{U}(\boldsymbol{p}) C_{\lambda=0}(P)\right]^{1 / 2}$, in which $\tilde{U}(\boldsymbol{p})=4 \pi / \boldsymbol{p}^{2}$ is the Coulomb interaction in the momentum representation and $C_{\lambda=0}(P)$ is evaluated from the connected density correlation function in the free system:

$$
\tilde{G}_{\lambda=0}^{(n)}\left(P_{1}, \ldots, P_{n-1}\right)=-2 \sum_{\sigma \in S_{n-1}} \int_{P^{\prime}} \prod_{k=0}^{n-1} \tilde{G}_{\mathrm{F}, 0}^{(2)}\left(\sum_{i=1}^{k} P_{\sigma(i)}+P^{\prime}\right),
$$

with the symmetric group $S_{n-1}$ of order $n-1$, the twopoint propagator of free fermions $\tilde{G}_{\mathrm{F}, 0}^{(2)}(P)=[i \omega-\xi(\boldsymbol{p})]^{-1}$, $\xi(\boldsymbol{p}):=\boldsymbol{p}^{2} / 2-p_{\mathrm{F}}^{2} / 2$, and the Fermi momentum $p_{\mathrm{F}}=$ $(9 \pi / 4)^{1 / 3} / r_{\mathrm{s}}$. 
Before ending the summary of the formalism, we comment on the behavior of Eq. (5) at the high-density limit $r_{\mathrm{s}} \rightarrow 0$. Through the use of the scaling behavior

$$
\begin{aligned}
& \left.\tilde{G}_{\lambda=0}^{(2)}(P)\right|_{r_{\mathrm{s}}}=\left.r_{\mathrm{s}}^{-1} \tilde{G}_{\lambda=0}^{(2)}(\bar{P})\right|_{r_{\mathrm{s}}=1}, \\
& \left.C_{\lambda=0}(P)\right|_{r_{\mathrm{s}}}=\left.C_{\lambda=0}(\bar{P})\right|_{r_{\mathrm{s}}=1},
\end{aligned}
$$

with the dimensionless momentum $\bar{P}=\left(r_{\mathrm{s}}^{2} \omega, r_{\mathrm{s}} \boldsymbol{p}\right)$, the expansion of Eq. (5) with respect to $r_{\mathrm{s}}$ is obtained. From the expansion, one finds that the exact behavior $\varepsilon_{\mathrm{corr}}^{\mathrm{GB}}\left(r_{\mathrm{s}}\right)$ is reproduced at $r_{\mathrm{s}} \rightarrow 0$.

Reduction of dimension of multiintegral. A difficulty in the three-dimensional system may be that the numerical evaluation of multiintegrals with respect to momenta is too costly to get $\varepsilon_{\text {corr }}\left(r_{\mathrm{s}}\right)$ for various $r_{\mathrm{s}}$. We find that this can be circumvented, since the dimension of the integral in Eq. (4) can be drastically reduced in an analytic manner.

Using the expression for $\tilde{G}_{\lambda=0}^{(n)}\left(P_{1}, \ldots, P_{n-1}\right)$ given in the sentence below Eq. (5) and performing the frequency integral, Eq. (4) becomes

$$
\begin{aligned}
C_{\lambda=0}(P)= & 2 \sum_{s=0,1}(-1)^{s+1} \int_{\boldsymbol{p}^{\prime}, \boldsymbol{p}^{\prime \prime}} \tilde{U}\left(\boldsymbol{p}^{\prime}-\boldsymbol{p}^{\prime \prime}-s \boldsymbol{p}\right) \theta_{\boldsymbol{p}^{\prime}} \theta_{\boldsymbol{p}^{\prime \prime}} \\
& \times\left[D\left(\omega, \boldsymbol{p}, \boldsymbol{p}^{\prime}-\boldsymbol{s} \boldsymbol{p}\right)-D\left(\omega, \boldsymbol{p}, \boldsymbol{p}^{\prime \prime}\right)\right]^{2},
\end{aligned}
$$

where

$$
D\left(\omega, \boldsymbol{p}, \boldsymbol{p}^{\prime}\right)=\left[i \omega-\xi\left(\boldsymbol{p}^{\prime}+\boldsymbol{p}\right)+\xi\left(\boldsymbol{p}^{\prime}\right)\right]^{-1},
$$

and $\theta_{\boldsymbol{p}}=\theta(-\xi(\boldsymbol{p}))$ with the Heaviside step function $\theta(x)$. By using $\tilde{U}(\boldsymbol{p})=\int d \boldsymbol{x} e^{i \boldsymbol{p} \cdot \boldsymbol{x}} /|\boldsymbol{x}|$ and employing the cylindrical coordinates in which the direction of the longitudinal axis $(z$ axis) is parallel to $\boldsymbol{p}$, Eq. (6) is rewritten as follows:

$$
\begin{aligned}
C_{\lambda=0}(P)= & \sum_{s=0,1} \frac{(-1)^{s+1}}{2 \pi^{3}} \int_{-p_{\mathrm{F}}}^{p_{\mathrm{F}}} d p_{z}^{\prime} \int_{-p_{\mathrm{F}}}^{p_{\mathrm{F}}} d p_{z}^{\prime \prime} P_{r}\left(p_{z}^{\prime}\right) P_{r}\left(p_{z}^{\prime \prime}\right) \\
& \times\left[D^{\prime}\left(\omega, p, p_{z}^{\prime}\right)-D^{\prime}\left(\omega, p, p_{z}^{\prime \prime}-s p\right)\right]^{2} \\
& \times I\left(P_{r}\left(p_{z}^{\prime}\right), P_{r}\left(p_{z}^{\prime \prime}\right),\left|p_{z}^{\prime}-p_{z}^{\prime \prime}+s p\right|\right) .
\end{aligned}
$$

Here, we have introduced $P_{r}\left(p_{z}\right)=\left(p_{\mathrm{F}}^{2}-p_{z}^{2}\right)^{1 / 2}$, $D^{\prime}\left(\omega, p, p_{z}^{\prime}\right)=\left(i \omega+p p_{z}^{\prime}+p^{2} / 2\right)^{-1}$, and

$$
I(a, b, c)=\int_{0}^{\infty} d r \frac{1}{r} J_{1}(a r) J_{1}(b r) K_{0}(c r),
$$

where $J_{1}(x)$ and $K_{0}(x)$ are the Bessel function of the first kind and the modified Bessel function of the second kind, respectively. Then, the integral in $I(a, b, c)$ can be performed analytically [31]:

$$
\begin{aligned}
I(a, b, c)= & l_{1}^{2}(a, b, c)+b^{2} \ln \left[1-\frac{l_{1}^{2}(a, b, c)}{b^{2}}\right] \\
& +c^{2} \ln \left[1-\frac{l_{1}^{2}(a, b, c)}{c^{2}}\right],
\end{aligned}
$$

where

$$
l_{1}(a, b, c)=\frac{1}{2}\left[\sqrt{(b+c)^{2}+a^{2}}-\sqrt{(b-c)^{2}+a^{2}}\right] .
$$

Finally, Eq. (7) together with Eq. (8) shows that only a double integral is required for the calculation of $C_{\lambda=0}(P)$. Needless

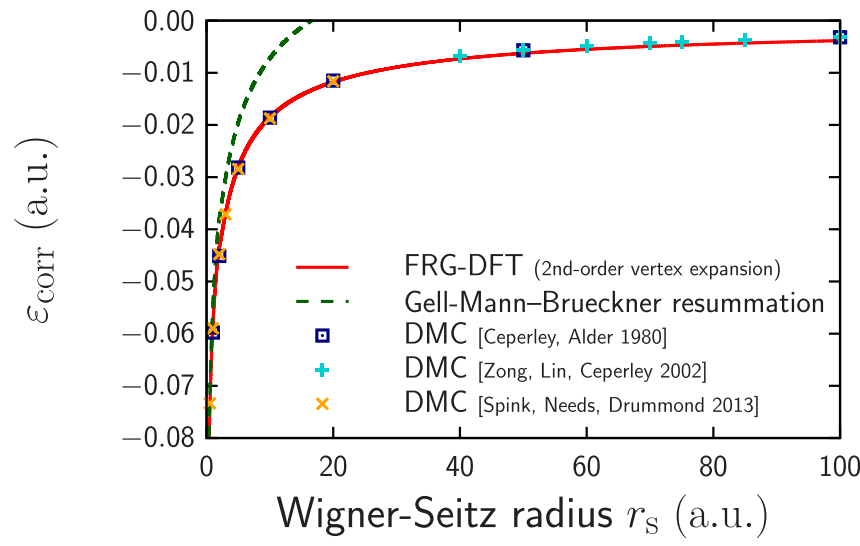

FIG. 1. Correlation energy par particle, $\varepsilon_{\text {corr }}$, of the 3DHEG derived by the FRG-DFT method as a function of $r_{\mathrm{s}}$. The results derived by the Gell-Mann-Brueckner resummation and the diffusion Monte Carlo (DMC) calculations are also shown. The DMC results are obtained by subtracting the kinetic and exchange energies [32,33] from the total energies given in Refs. [28-30].

to say, the isotropy reduces the dimension of the integral in Eq. (5).

Results of the correlation energy. The reduction of the dimension of the integral saves the time for the numerical calculation and enables one to obtain $\varepsilon_{\text {corr }}$ for many $r_{\mathrm{s}}$. The calculation was carried out on 65536 grid points with the logarithmic mesh in $r_{\mathrm{s}} \in\left[10^{-6}\right.$ a.u., 100 a.u.).

Figure 1 shows $\varepsilon_{\text {corr }}\left(r_{\mathrm{s}}\right)$ obtained by the FRG-DFT together with $\varepsilon_{\mathrm{corr}}^{\mathrm{GB}}\left(r_{\mathrm{s}}\right)$ and the DMC results [28-30]. As expected from our analytical discussion, one can see the FRG-DFT result reproduces the exact behavior at the high-density limit given by $\varepsilon_{\text {corr }}^{\mathrm{GB}}\left(r_{\mathrm{s}}\right)$. The FRG-DFT result is also consistent with the DMC results in a wide range of $r_{\mathrm{s}}$, and in particular the agreement becomes better as the density increases: The deviations from the results in Ref. [28] are about $2.0 \%$ at $r_{\mathrm{s}}=1$ a.u., $6.7 \%$ at $r_{\mathrm{s}}=50$ a.u., and $17 \%$ at $r_{\mathrm{s}}=100$ a.u.

Now we have shown that $\varepsilon_{\text {corr }}\left(r_{\mathrm{s}}\right)$ can be obtained in the framework of the FRG-DFT and becomes accurate as the density increases, which is one of the main results of this Letter. In the remaining part, we attempt a construction of the EDF by use of our $\varepsilon_{\text {corr }}\left(r_{\mathrm{s}}\right)$.

Construction of the energy density functional. Since $\varepsilon_{\text {corr }}$ are obtained very densely for various $r_{\mathrm{s}}$ in our scheme, we can construct the LDA EDF $E_{\text {corr }}^{\mathrm{LDA}}[\rho]=\int \boldsymbol{d} \boldsymbol{x} \rho(\boldsymbol{x}) \varepsilon_{\mathrm{corr}}\left(r_{\mathrm{s}}(\rho(\boldsymbol{x}))\right)$ without any fitting for physically relevant densities. This is in sharp contrast to other conventional EDFs, such as VWN [34], PZ81 [35], and PW92 [36], which are determined by fitting the few DMC data obtained by Ceperley and Alder [28].

Our functional, which is referred to as the FRG-numericaltable functional (FRG-NT), is constructed as follows: In $r_{\mathrm{s}} \in\left[10^{-6}\right.$ a.u., 100 a.u. $), \varepsilon_{\text {corr }}\left(r_{\mathrm{s}}\right)$ are determined by the interpolation of the FRG-DFT data. For simplicity, we employ the linear interpolation; the results hardly depend on the choice of the interpolation function. In $r_{\mathrm{s}}<$ $10^{-6}$ a.u., $\varepsilon_{\text {corr }}\left(r_{\mathrm{s}}\right)$ is substituted by $\varepsilon_{\mathrm{corr}}^{\mathrm{GB}}\left(r_{\mathrm{s}}\right)$. The FRG-DFT data are extrapolated to $r_{\mathrm{s}} \geqslant 100 \mathrm{a} . \mathrm{u}$. by a fitting function $\varepsilon_{\text {corr }}\left(r_{\mathrm{s}}\right)=\gamma /\left(1+\beta_{1} \sqrt{r_{\mathrm{s}}}+\beta_{2} r_{\mathrm{s}}\right)$ [35]. The fitting parameters are chosen to be $\gamma=0.0378052, \beta_{1}=-0.801035$, and 
TABLE I. Parameters for FRG-PZ. For comparison, the parameters of PZ81 [35] are also shown. All the data are shown in the Hartree atomic units.

\begin{tabular}{lrc}
\hline \hline & PZ81 [35] & \multicolumn{1}{c}{ FRG-PZ } \\
\hline$C$ & 0.0020 & 0.00173055 \\
$D$ & -0.0116 & -0.0100569 \\
$\gamma$ & -0.1423 & -0.175617 \\
$\beta_{1}$ & 1.0529 & 1.67669 \\
$\beta_{2}$ & 0.3334 & 0.348219 \\
\hline
\end{tabular}

$\beta_{2}=-0.0306778$, which are obtained by fitting the data in 95 a.u. $<r_{\mathrm{s}}<100$ a.u.

A remark is in order here: The evaluation of $\varepsilon_{\text {corr }}^{\prime}\left(r_{\mathrm{s}}\right)=d \varepsilon_{\text {corr }}\left(r_{\mathrm{s}}\right) / d r_{\mathrm{s}}$ appearing in the $\mathrm{KS}$ potential $\delta E_{\text {corr }}^{\mathrm{LDA}}[\rho] / \delta \rho(\boldsymbol{x})=\left[\varepsilon_{\text {corr }}\left(r_{\mathrm{s}}\right)-\left(r_{\mathrm{s}} / 3\right) \varepsilon_{\text {corr }}^{\prime}\left(r_{\mathrm{s}}\right)\right]_{r_{\mathrm{s}}=r_{\mathrm{s}}(\rho(\boldsymbol{x}))}$ with the numerical differentiation may cause numerical errors. We evade this by performing the analytic differentiation of Eq. (5):

$$
\varepsilon_{\text {corr }}^{\prime}\left(r_{\mathrm{s}}\right)=\frac{1}{2 n_{e} r_{\mathrm{s}}} \int_{P}\left[g\left(A_{P}, B_{P}\right)-2 \ln f\left(A_{P}, B_{P}\right)\right],
$$

where $g(x, y)=x+(x \cosh y+y \sinh y) / f(x, y)$. To perform the differentiation, we have used $\left.\tilde{G}_{\lambda=0}^{(2)}(P)\right|_{r_{\mathrm{s}}}=$ $\left.r_{\mathrm{s}}^{-1} \tilde{G}_{\lambda=0}^{(2)}(\bar{P})\right|_{r_{\mathrm{s}}=1},\left.\quad C_{\lambda=0}(P)\right|_{r_{\mathrm{s}}}=\left.C_{\lambda=0}(\bar{P})\right|_{r_{\mathrm{s}}=1}$ and rewritten Eq. (5) in terms of $r_{\mathrm{s}}$ and quantities independent of $r_{\mathrm{s}}$. We calculate $\varepsilon_{\text {corr }}^{\prime}\left(r_{\mathrm{s}}\right)$ on the same grid for $r_{\mathrm{s}}$ as $\varepsilon_{\text {corr }}\left(r_{\mathrm{s}}\right)$, and determine $\varepsilon_{\text {corr }}^{\prime}\left(r_{\mathrm{s}}\right)$ for arbitrary $r_{\mathrm{s}}$ in the same manner as $\varepsilon_{\text {corr }}\left(r_{\mathrm{s}}\right)$.

Additionally, we prepare a functional, which we name FRG-PZ, by fitting the FRG-DFT data with the same function as PZ81:

$$
\varepsilon_{\text {corr }}\left(r_{\mathrm{s}}\right)= \begin{cases}A \ln r_{\mathrm{s}}+B+C r_{\mathrm{s}} \ln r_{\mathrm{s}}+D r_{\mathrm{s}} & r_{\mathrm{s}}<1 \text { a.u. } \\ \gamma /\left(1+\beta_{1} \sqrt{r_{\mathrm{s}}}+\beta_{2} r_{\mathrm{s}}\right) & r_{\mathrm{s}} \geqslant 1 \text { a.u. }\end{cases}
$$

for the purpose of comparing our functionals to PZ81 and discussing the origin of the deviation between EDFs. Here, $A=0.0311$ and $B=-0.0480$ reproduce $\varepsilon_{\text {corr }}^{\mathrm{GB}}\left(r_{\mathrm{s}}\right)$ at $r_{\mathrm{s}} \rightarrow 0$. The remaining parameters $C, D, \gamma, \beta_{1}$, and $\beta_{2}$ are related to each other through the continuum conditions for $\varepsilon_{\text {corr }}\left(r_{\mathrm{s}}\right)$ and $\varepsilon_{\text {corr }}^{\prime}\left(r_{\mathrm{s}}\right)$ at $r_{\mathrm{s}}=1$ a.u:

$$
\begin{aligned}
\gamma & =\left(1+\beta_{1}+\beta_{2}\right)(B+D), \\
\beta_{2} & =-\frac{2(A+C)\left(1+\beta_{1}\right)+B \beta_{1}+2 D+3 \beta_{1} D}{2(A+B+C+2 D)} .
\end{aligned}
$$

Table I lists the values of the parameters obtained by the fitting with the conditions Eqs. (11a) and (11b).

Benchmark test of the functionals. We apply our EDF to the $\mathrm{KS}$ calculation of the ground-state energies of noble gas atoms and compare with other conventional EDFs, such as VWN [34], PZ81 [35], PW92 [36], Chachiyo [37], revChachiyo [38], and GGA-PBE [39]. The numerical calculation was carried out by use of ADPACK [40].

Figure 2 shows the ground-state energies $E^{\mathrm{gs}}$ of $\mathrm{Ne}, \mathrm{Ar}$, $\mathrm{Kr}, \mathrm{Xe}$, and $\mathrm{Rn}$ atoms obtained by each EDF as ratios to the results of PZ81 $E_{\mathrm{PZ} 81}^{\mathrm{gs}}$. One can see that the functionals constructed from the FRG-DFT show comparable results to those of other LDA EDFs for every atom. On the other hand,

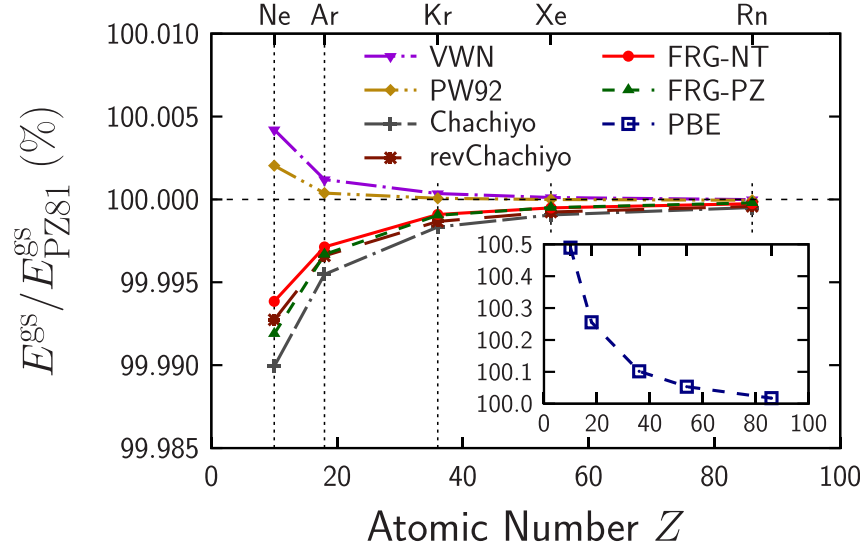

FIG. 2. Ratios of the ground-state energies to that given by PZ81 shown as functions of the atomic number $Z$.

the GGA-PBE results are quite different from those of the LDA EDFs. This suggests that the results of our functional reside close to those of the LDA EDF determined by the exact correlation energy per particle for 3DHEG $\varepsilon_{\text {corr }}^{\text {exact }}\left(r_{\mathrm{s}}\right)$ as much as other conventional LDA EDFs, and the differences between our functional and other LDA EDFs are insignificant for the accuracy in comparison with the effect of the ignorance of the gradient.

Origins of the difference among functionals. To further understand the difference between LDA EDFs, we investigate the difference of the energy with fixing the density, inspired by the notion of the functional-driven error [41]. Figure 3 shows $\Delta E_{\mathrm{F}}^{\mathrm{gs}}=E\left[\rho_{\mathrm{gs}}^{\mathrm{PZ} 81}\right]-E_{\mathrm{PZ} 81}\left[\rho_{\mathrm{gs}}^{\mathrm{PZ} 81}\right]$, i.e., the deviation of each $\operatorname{EDF} E[\rho]$ from PZ81 $E_{\mathrm{PZ} 81}[\rho]$ at $\rho(\boldsymbol{x})=\rho_{\mathrm{gs}}^{\mathrm{PZ} 81}(\boldsymbol{x})$ being the ground-state density obtained by PZ81. One can see that the deviations among EDFs are comparable even at the same density. In the case of LDA, this deviation originates from $\varepsilon_{\text {corr }}\left(r_{\mathrm{s}}\right)$, the error of which is expected to be attributed to two parts: the reference-driven error and the fitting-driven error, i.e., the errors caused by the choice of the reference data and the fitting functions, respectively. By recasting the difference between EDFs in terms of these two errors, further

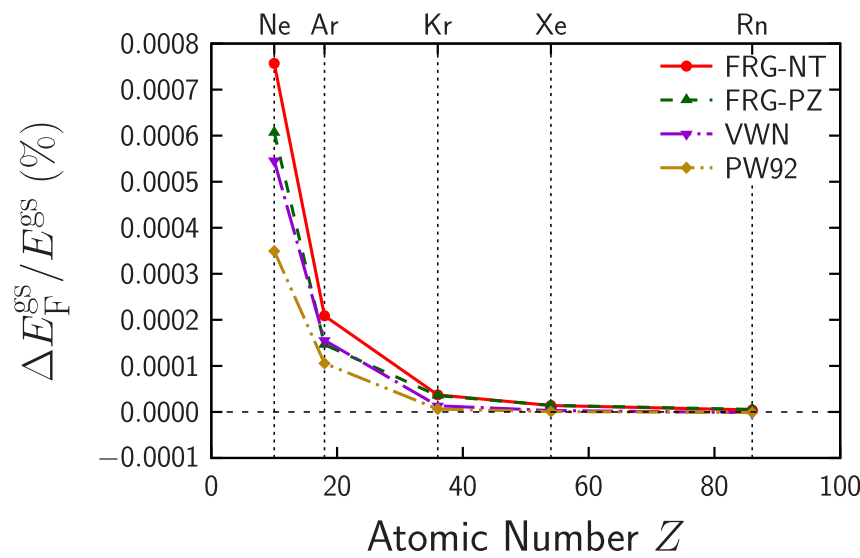

FIG. 3. Deviations of energies from that given by PZ81 at a fixed density $\rho_{\mathrm{gs}}^{\mathrm{PZ}}(\boldsymbol{x})$ for each EDF shown as the ratio to the ground-state energy obtained by the EDF. 


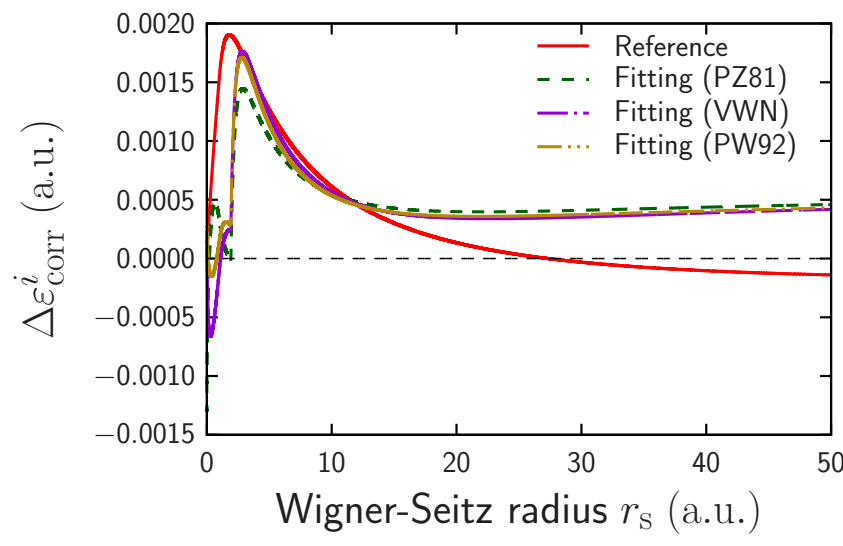

FIG. 4. Dependence on $r_{\mathrm{s}}$ of $\Delta \varepsilon_{\text {corr,ref }}^{\mathrm{FRG}}-\Delta \varepsilon_{\text {corr,ref }}^{\mathrm{DMC}}$ (Reference), $\Delta \varepsilon_{\text {corr,fit }}^{\mathrm{PZ81}}($ Fitting $(\mathrm{PZ81})), \Delta \varepsilon_{\text {corr,fit }}^{\mathrm{VWN}}($ Fitting $(\mathrm{VWN}))$, and $\Delta \varepsilon_{\text {corr,fit }}^{\mathrm{PW} 92}$ (Fitting (PW92)).

understanding of the origin of $\Delta E_{\mathrm{F}}^{\mathrm{gs}}$ shown in Fig. 3 will be obtained.

We roughly assume that $\varepsilon_{\text {corr }}^{i}\left(r_{\mathrm{s}}\right)$ standing for $\varepsilon_{\text {corr }}\left(r_{\mathrm{s}}\right)$ used for functional $i$ (=VWN, PZ81, PW92, FRG-NT, FRG-PZ) is written as

$$
\varepsilon_{\text {corr }}^{i}\left(r_{\mathrm{s}}\right)=\varepsilon_{\text {corr }}^{\text {exact }}\left(r_{\mathrm{s}}\right)+\Delta \varepsilon_{\text {corr,ref }}^{i}\left(r_{\mathrm{s}}\right)+\Delta \varepsilon_{\text {corr, fit }}^{i}\left(r_{\mathrm{s}}\right),
$$

with the reference-driven error $\Delta \varepsilon_{\text {corr,ref }}^{i}\left(r_{\mathrm{s}}\right)$ and fitting-driven error $\Delta \varepsilon_{\text {corr,fit }}^{i}\left(r_{\mathrm{s}}\right)$. Since FRG-NT does not rely on any fitting function,

$$
\Delta \varepsilon_{\text {corr,fit }}^{\text {FRG-NT }} \equiv 0 .
$$

The fact that the same fitting scheme is employed for FRG-PZ and PZ81 leads to

$$
\Delta \varepsilon_{\text {corr,fit }}^{\mathrm{FRG}}\left(r_{\mathrm{s}}\right)=\Delta \varepsilon_{\text {corr,fit }}^{\mathrm{PZ} 81}\left(r_{\mathrm{s}}\right) .
$$

We also have

$$
\Delta \varepsilon_{\text {corr,ref }}^{i}\left(r_{\mathrm{s}}\right)= \begin{cases}\Delta \varepsilon_{\text {corr,ref }}^{\mathrm{DMC}}\left(r_{\mathrm{s}}\right) & (i=\mathrm{VWN}, \mathrm{PZ} 81, \mathrm{PW} 2), \\ \Delta \varepsilon_{\mathrm{corr}, \mathrm{ref}}^{\mathrm{FRG}}\left(r_{\mathrm{s}}\right) & (i=\text { FRG-NT, FRG-PZ }),\end{cases}
$$

where $\Delta \varepsilon_{\text {corr,ref }}^{\mathrm{DMC}}\left(r_{\mathrm{s}}\right)$ and $\Delta \varepsilon_{\text {corr,ref }}^{\mathrm{FRG}}\left(r_{\mathrm{s}}\right)$ are errors stemming from the choice of DMC and FRG data, respectively. By use of these conditions, $\Delta \varepsilon_{\text {corr,fit }}^{i}\left(r_{\mathrm{s}}\right)$ and $\Delta \varepsilon_{\text {corr,ref }}^{\mathrm{FRG}}\left(r_{\mathrm{s}}\right)-\Delta \varepsilon_{\text {corr,ref }}^{\mathrm{DMC}}\left(r_{\mathrm{s}}\right)$ are estimated from $\varepsilon_{\mathrm{corr}}^{i}\left(r_{\mathrm{s}}\right)$.
Figure 4 shows $\Delta \varepsilon_{\text {corr,fit }}^{i}\left(r_{\mathrm{s}}\right)$ and $\Delta \varepsilon_{\text {corr,ref }}^{\mathrm{FRG}}\left(r_{\mathrm{s}}\right)-$ $\Delta \varepsilon_{\text {corr,ref }}^{\text {DMC }}\left(r_{\mathrm{s}}\right)$. In $r_{\mathrm{s}} \gtrsim 10$ a.u., the use of fitting affects the value of $\varepsilon_{\text {corr }}\left(r_{\mathrm{s}}\right)$ more than the choice of the reference data does when these quantities have comparable magnitude in $r_{\mathrm{s}} \lesssim 10$ a.u. This may explain why the comparable results for each functional are obtained in Fig. 3, since $r_{\mathrm{s}} \lesssim 10 \mathrm{a}$.u. is physically relevant for atoms.

Conclusion. We presented an ab initio construction of the energy density functional (EDF) for three-dimensional electron systems using the functional-renormalization-groupaided density functional theory (FRG-DFT). The derived correlation energies of the homogeneous electron gas agree with the Monte Carlo results in a wide range of densities, reproducing the exact behavior given by the Gell-MannBrueckner resummation at the high-density limit. Using the FRG-DFT data obtained densely for various densities, we construct the EDF in the local density approximation (LDA) without using any fitting function for physically relevant densities. Applied to the KS calculation of the ground-state energies of the noble gas atoms, our functional shows comparable results to other conventional ones in LDA. Our results show that FRG-DFT can become a practical method contributing to the nonempirical construction of EDFs of realistic quantum many-body systems.

Although we have focused on the case of LDA in this Letter, our formalism is also applicable for the construction of EDFs incorporating the effect of the gradient of density. There are some technical ideas to realize the inclusion of gradient effects, such as the use of the weighted density approximation or the derivative expansion, which has been developed in the context of FRG. Based on these ideas, we believe that the construction of EDFs beyond LDA without any empirical parameter is achievable. Our formalism and procedure can also be naturally extended to the case of constructing EDFs in the local spin density approximation, which will be presented in a forthcoming paper.

Acknowledgments. The authors acknowledge Osamu Sugino for valuable comments on the manuscript and Ryosuke Akashi, Le Minh Cristian, Haruki Kasuya, Teiji Kunihiro, Peter Maksym, Shinji Tsuneyuki, and Kenichi Yoshida for fruitful discussions. T.Y. was supported by the Grants-in-Aid for JSPS fellows (Grant No. 20J00644). T.N. was supported by the Grants-in-Aid for JSPS fellows (Grant No. 19J20543). Numerical computation in this work was carried out on Cray $\mathrm{XC}$ at the Yukawa Institute Computer Facility and cluster computers at the RIKEN iTHEMS program.
[1] P. Hohenberg and W. Kohn, Inhomogeneous electron gas, Phys. Rev. 136, B864 (1964).

[2] W. Kohn and L. J. Sham, Self-consistent equations including exchange and correlation effects, Phys. Rev. 140, A1133 (1965).

[3] J. P. Perdew and K. Schmidt, Jacob's ladder of density functional approximations for the exchange-correlation energy, AIP Conf. Proc. 577, 1 (2001).
[4] M. G. Medvedev, I. S. Bushmarinov, J. Sun, J. P. Perdew, and K. A. Lyssenko, Density functional theory is straying from the path toward the exact functional, Science 355, 49 (2017).

[5] K. P. Kepp, Comment on "density functional theory is straying from the path toward the exact functional", Science 356, 496 (2017).

[6] J. Polonyi and K. Sailer, Effective actions and the density functional theory, Phys. Rev. B 66, 155113 (2002). 
[7] A. Schwenk and J. Polonyi, Towards density functional calculations from nuclear forces, in 32nd International Workshop on Gross Properties of Nuclei and Nuclear Excitation: Probing Nuclei and Nucleons with Electrons and Photons (Hirschegg 2004), Hirschegg, Austria, January 11-17, 2004 (2004), pp. 273-282, arXiv:nucl-th/0403011.

[8] F. J. Wegner and A. Houghton, Renormalization Group Equation for Critical Phenomena, Phys. Rev. A 8, 401 (1973).

[9] K. G. Wilson and J. Kogut, The renormalization group and the $\varepsilon$ expansion, Phys. Rep. 12, 75 (1974).

[10] J. Polchinski, Renormalization and effective lagrangians, Nucl. Phys. B 231, 269 (1984).

[11] C. Wetterich, Exact evolution equation for the effective potential, Phys. Lett. B 301, 90 (1993).

[12] J. Berges, N. Tetradis, and C. Wetterich, Nonperturbative renormalization flow in quantum field theory and statistical physics, Phys. Rep. 363, 223 (2002).

[13] J. M. Pawlowski, Aspects of the functional renormalisation group, Ann. Phys. 322, 2831 (2007).

[14] H. Gies, Introduction to the functional RG and applications to Gauge theories, in Renormalization Group and Effective Field Theory Approaches to Many-Body Systems, edited by A. Schwenk and J. Polonyi (Springer, Berlin, Heidelberg, 2012), pp. 287-348.

[15] W. Metzner, M. Salmhofer, C. Honerkamp, V. Meden, and K. Schönhammer, Functional renormalization group approach to correlated fermion systems, Rev. Mod. Phys. 84, 299 (2012).

[16] N. Dupuis, L. Canet, A. Eichhorn, W. Metzner, J. M. Pawlowski, M. Tissier, and N. Wschebor, The nonperturbative functional renormalization group and its applications, Phys. Rep. (2021), doi:10.1016/j.physrep.2021.01.001.

[17] R. Fukuda, T. Kotani, Y. Suzuki, and S. Yokojima, Density functional theory through legendre transformation, Prog. Theor. Phys. 92, 833 (1994).

[18] R. Fukuda, M. Komachiya, S. Yokojima, Y. Suzuki, K. Okumura, and T. Inagaki, Novel use of Legendre transformation in field theory and many particle systems: On-shell expansion and inversion method, Prog. Theor. Phys. Suppl. 121, 1 (1995).

[19] M. Valiev and G. W. Fernando, Generalized Kohn-Sham Density-Functional Theory via Effective Action Formalism, (1997), arXiv:cond-mat/9702247.

[20] S. Kemler and J. Braun, Towards a renormalization group approach to density functional theory-general formalism and case studies, J. Phys. G 40, 085105 (2013).

[21] S. Kemler, M. Pospiech, and J. Braun, Formation of selfbound states in a one-dimensional nuclear model-a renormalization group based density functional study, J. Phys. G 44, 015101 (2017).

[22] H. Liang, Y. Niu, and T. Hatsuda, Functional renormalization group and Kohn-Sham scheme in density functional theory, Phys. Lett. B 779, 436 (2018).

[23] T. Yokota, K. Yoshida, and T. Kunihiro, Functional renormalization-group calculation of the equation of state of one-dimensional uniform matter inspired by the hohenberg-kohn theorem, Phys. Rev. C 99, 024302 (2019).

[24] T. Yokota and T. Naito, Functional-renormalization-group aided density functional analysis for the correlation energy of the two-dimensional homogeneous electron gas, Phys. Rev. B 99, 115106 (2019).

[25] T. Yokota, K. Yoshida, and T. Kunihiro, Ab initio description of excited states of 1D uniform matter with the Hohenberg-Kohntheorem-inspired functional-renormalization-group method, Prog. Theor. Exp. Phys. 2019, 011 D01 (2019).

[26] T. Yokota, H. Kasuya, K. Yoshida, and T. Kunihiro, Microscopic derivation of density functional theory for superfluid systems based on effective action formalism, Prog. Theor. Exp. Phys. 2021, 013 A03 (2020).

[27] M. Gell-Mann and K. A. Brueckner, Correlation energy of an electron gas at high density, Phys. Rev. 106, 364 (1957).

[28] D. M. Ceperley and B. J. Alder, Ground State of the Electron Gas by a Stochastic Method, Phys. Rev. Lett. 45, 566 (1980).

[29] F. H. Zong, C. Lin, and D. M. Ceperley, Spin polarization of the low-density three-dimensional electron gas, Phys. Rev. E 66, 036703 (2002).

[30] G. G. Spink, R. J. Needs, and N. D. Drummond, Quantum Monte Carlo study of the three-dimensional spin-polarized homogeneous electron gas, Phys. Rev. B 88, 085121 (2013).

[31] D. Zwillinger, Table of Integrals, Series, and Products (Academic Press, Cambridge, MA, 2014).

[32] J. C. Slater, A simplification of the Hartree-Fock method, Phys. Rev. 81, 385 (1951).

[33] G. D. Mahan, Many-Particle Physics, Physics of Solids and Liquids (Springer, New York, 2000).

[34] S. H. Vosko, L. Wilk, and M. Nusair, Accurate spin-dependent electron liquid correlation energies for local spin density calculations: a critical analysis, Can. J. Phys. 58, 1200 (1980).

[35] J. P. Perdew and A. Zunger, Self-interaction correction to density-functional approximations for many-electron systems, Phys. Rev. B 23, 5048 (1981).

[36] J. P. Perdew and Y. Wang, Accurate and simple analytic representation of the electron-gas correlation energy, Phys. Rev. B 45, 13244 (1992).

[37] T. Chachiyo, Communication: Simple and accurate uniform electron gas correlation energy for the full range of densities, J. Chem. Phys. 145, 021101 (2016).

[38] V. V. Karasiev, Comment on "Communication: Simple and accurate uniform electron gas correlation energy for the full range of densities" [J. Chem. Phys. 145, 021101 (2016)], J. Chem. Phys. 145, 157101 (2016).

[39] J. P. Perdew, K. Burke, and M. Ernzerhof, Generalized Gradient Approximation Made Simple, Phys. Rev. Lett. 77, 3865 (1996).

[40] T. Ozaki, H. Kino, H. Kawai, and M. Toyoda, ADPACK Ver.2.2, http://www.openmx-square.org/adpack_man2.2/ (2011).

[41] M.-C. Kim, E. Sim, and K. Burke, Understanding and Reducing Errors in Density Functional Calculations, Phys. Rev. Lett. 111, 073003 (2013). 